\title{
Cross-sectional associations between eating occasions, meals, and snacks with blood lipids in a population-based cohort
}

\author{
M.A.H. Lentjes ${ }^{1}$, L.M. Oude Griep ${ }^{2}$, R.H. Keogh ${ }^{3}$, A.A. Mulligan ${ }^{1}$ and K.T. Khaw ${ }^{4}$ \\ ${ }^{1}$ University of Cambridge, Dept of Public Health \& Primary Care, Cambridge CB1 8RN, \\ ${ }^{2}$ Imperial College London, \\ ${ }^{3}$ Dept of Epidemiology and Biostatistics, London W2 1PG, London School of Hygiene and Tropical Medicine, London \\ WCIE 7 HT and \\ ${ }^{4}$ University of Cambridge, Clinical Gerontology Unit, Cambridge CB2 $2 Q Q$
}

The frequency and dispersion of eating occasions (EOs), including both meals and snacks, over the day has been associated with cardiovascular health ${ }^{(1,2)}$. Definitions of meals and snacks vary by dietary assessment methods and studies, which hamper comparisons between studies $^{(3)}$. The aims were (i) to compare various definitions of EOs, meals and snacks from a pre-structured 7-day diet diary (7dDD) and (ii) to examine their associations with blood lipids, while considering time-of-day variation of these biomarkers.

The Norfolk-based European Prospective Investigation into Cancer and Nutrition (EPIC-Norfolk) recruited men and women, aged 39-79 y from GP practices between 1993-1998(4). Anthropometry was measured at health visits at which point non-fasting blood samples were taken and serum cholesterol and triglyceride (TG) concentrations determined. Participants completed a 7dDD. Each of eight recording sections of each day were classified as an EO based on the reported energy intake in each section $(>0 \mathrm{~kJ}$ or $>210$ $\mathrm{kJ})$. Additionally, if a recording section contained $\geq 15 \%$ of the daily energy intake, the section was defined as a meal, otherwise as a snack $(>0-<15 \%)$. We then calculated the mean number of EOs, meals, and snacks per day over the number of recording days for every individual. We excluded those using lipid lowering medication or who reported illness or nightshifts during the $7 \mathrm{dDD}$. Analysis of variance was used to investigate associations of EO, meal, and snack frequency with lipid concentrations, adjusted for: hour of blood sampling, hours fasted, season, sex, age, physical activity, smoking, energy intake (higher eating frequency therefore indicate smaller EO), alcohol, education and BMI $(\mathrm{N}=22,068)$. TGs were log-transformed. Significant contributions to the model were determined by the F-test $(\mathrm{P}<0.05)$. Back-transformed adjusted means were graphed according to time-of-day of blood sampling (08:00-19:00).

The mean number of EOs/d was 6.2 if $\mathrm{EO}>0 \mathrm{~kJ}$ and 4.6 if $\mathrm{EO}>210 \mathrm{~kJ}$. The mean number of meals/d was 2.7 and snacks 1.9 (correlation $-0 \cdot 33$ ). EOs based on $>0$ and $>210 \mathrm{~kJ}$ were not significantly associated with cholesterol concentrations in an adjusted model. In a model with both meals and snacks, only meal frequency was associated with lower cholesterol $(\mathrm{P}<0.001)$. TG concentrations were higher as the day progressed. The associations with TG for mutually adjusted meal $(\mathrm{P}<0 \cdot 001)$ and snack frequency $(\mathrm{P}=0.021)$ were stronger than for any EO frequency, since meals were more strongly associated with higher TG than snacks. No interaction was found between meals/snacks in their associations with cholesterol or TG.
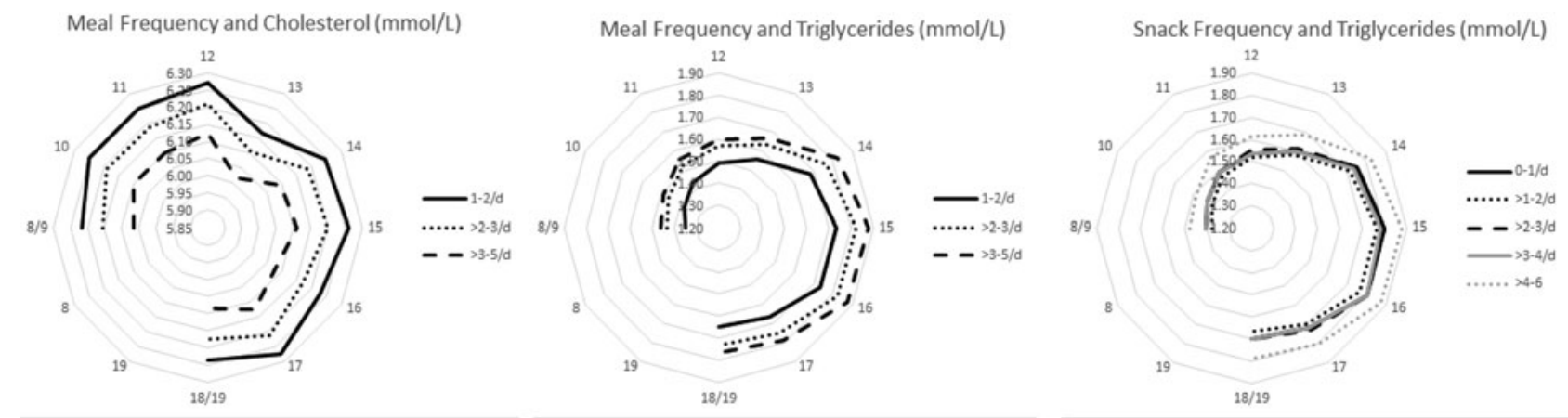

Not only EO frequency ${ }^{(2)}$, but also EO size (meal vs. snack) was important in the association with lipid biomarkers; since lower frequency of EOs and snacks, but mainly of meals, was associated with lower TG concentrations. Confounding factors such as the time of eating, size of the meal or the diet quality of meals $v s$. snacks might play a role.

1. St-Onge M-P, Ard J, Baskin ML et al. (2017) Circulation 135, e96-e121.

2. Titan SM, Bingham S, Welch A et al. (2001) BMJ 323, 1286-8.

3. Leech RM, Worsley A, Timperio A et al. (2015) Nutr Res Rev 28, 1-21.

4. Day N, Oakes S, Luben R et al. (1999) Br J Cancer 80, Suppl. 1, 95-103. 